\title{
Papillary muscle relocation with a multiloop suture: A proposed surgical technique for ischemic mitral regurgitation
}

\author{
Lawrence Torkan, BSc, ${ }^{\mathrm{a}, \mathrm{b}}$ Maria Theresa Servito, BHSc, ${ }^{\mathrm{a}}$ and Gianluigi Bisleri, MD, FRCSC, ${ }^{\mathrm{a}}$ Kingston, \\ Ontario, Canada
}

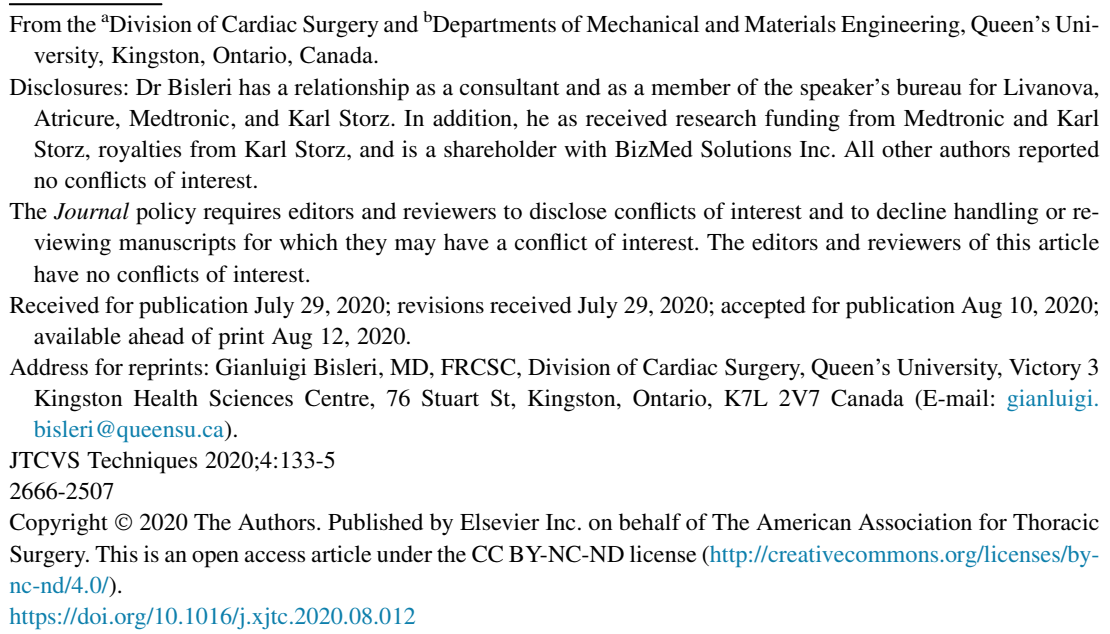

Video clip is available online.

Ischemic mitral regurgitation (IMR), a subtype of functional MR, occurs when mitral valve leaflets cannot adequately coapt in the absence of structural abnormalities. Functional MR is associated with increased morbidity and mortality. ${ }^{1}$ Ischemia causes left ventricular remodeling, annular dilatation, and poor leaflet coaptation. Treatment involves revascularization via coronary artery bypass grafting (CABG) and mitral valve repair via restrictive annuloplasty (RA). Recurrence of MR is still seen in up to $58.8 \%$ of patients at 2 years postoperatively. ${ }^{2}$ In most cases, persistent posterior leaflet tethering leads to poorly sustained coaptation. Papillary muscle relocation (PMR) strategies have been developed to adjust the subvalvular apparatus as an adjunct to RA, showing promising results in reducing MR recurrence. ${ }^{3}$ We present an innovative modification of the original PMR technique using a multiloop suture to treat moderate to severe IMR.

\section{SURGICAL TECHNIQUE}

Video 1 shows the preparation and application of the multi-loop. Each end of a polytetrafluoroethylene (PTFE)

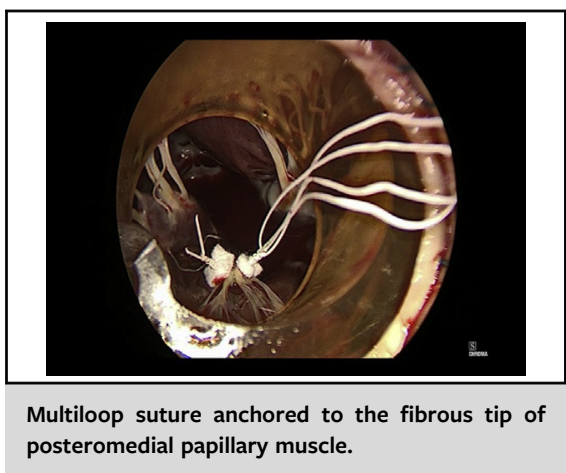

CENTRAL MESSAGE

We present a novel papillary muscle relocation technique us-

ing a multiloop technique to

address the subvalvular appa-

ratus in functional mitral

regurgitation.

See Commentaries on pages 136 and 138 .

suture (CV-4 Gore-Tex; WL Gore \& Associates Inc, Newark, Del) is passed through a PTFE felt pledget, creating a loop to which additional sutures can attach (Figure 1). Typically, 2 sutures are passed through the loop, tied tightly with multiple knots, and can slide along the primary suture. Transesophageal echocardiography is used to confirm the absence of leaflet abnormalities. Median sternotomy was performed. CABG was performed in a conventional fashion. Upon accessing the left atrium, an inspection confirms the echocardiographic findings regarding leaflet tethering, which typically occurs at the level of P2 and P3. The free ends of the loop are anchored to the fibrous tip of the posteromedial papillary muscle (PPM), which was buttressed with a pledget. PFTE sutures attached to the loop are passed through the P2 and P3 aspects of the annulus, from ventricular to the atrial side. RA is performed with a complete rigid ring (Profile 3D, Medtronic, Minneapolis, Minn) 2 sizes down from obtained measurements. Neochord lengths are then adjusted. The PPM is relocated to establish adequate coaptation at the annular plane. Saline 


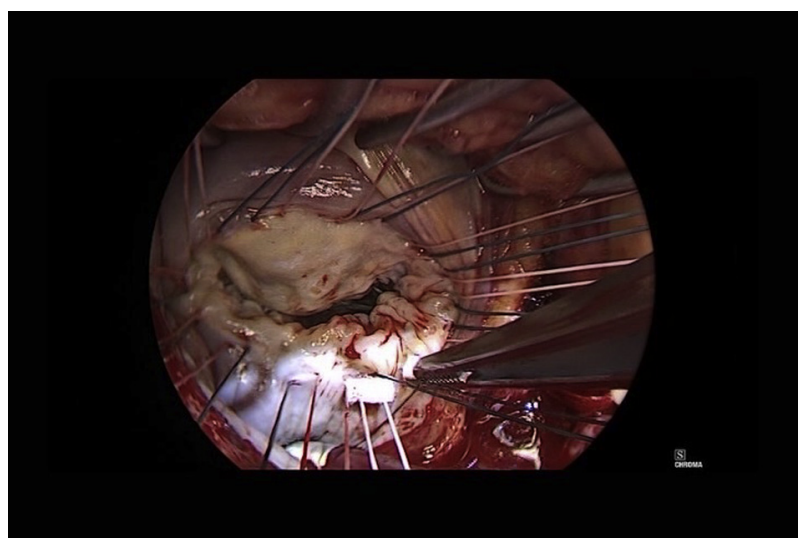

VIDEO 1. This video demonstrates the preparation of a multiloop suture for the purpose of papillary muscle relocation (PMR) to treat ischemic mitral regurgitation (IMR). Two polytetrafluoroethylene (PTFE) sutures (CV-4 Gore-Tex; WL Gore \& Associates Inc, Newark, Del) were tied to the end of a third to form the multiloop. The free ends of the loop were buttressed with a pledget and passed through the fibrous tip of the posteromedial papillary muscle (PPM). Neochordae were passed through P2 and P3 and ring annuloplasty (RA) followed. Valve competency was evaluated by manipulating the free ends of the neochordae while applying a water test. Transesophageal echocardiography (TEE) validated the quality of the repair. Video available at: https://www.jtcvs.org/article/S2666-2507 (20)30387-4/fulltext.

is injected into the left ventricular cavity to estimate neochord lengths and leaflet coaptation (Figure 2). Video 1 depicts that the progressive adjustment of chordal length allows visual appreciation of complete disappearance of regurgitation. Neochordae are deemed appropriate if MR is absent upon visual inspection; excessive traction may lead to posterior leaflet prolapse. Validating the absence of residual MR is required by transesophageal echocardiography once the patient has been weaned from cardiopulmonary bypass.

This technique was performed on 3 patients, all of whom provided informed consent (Table 1); no complications or

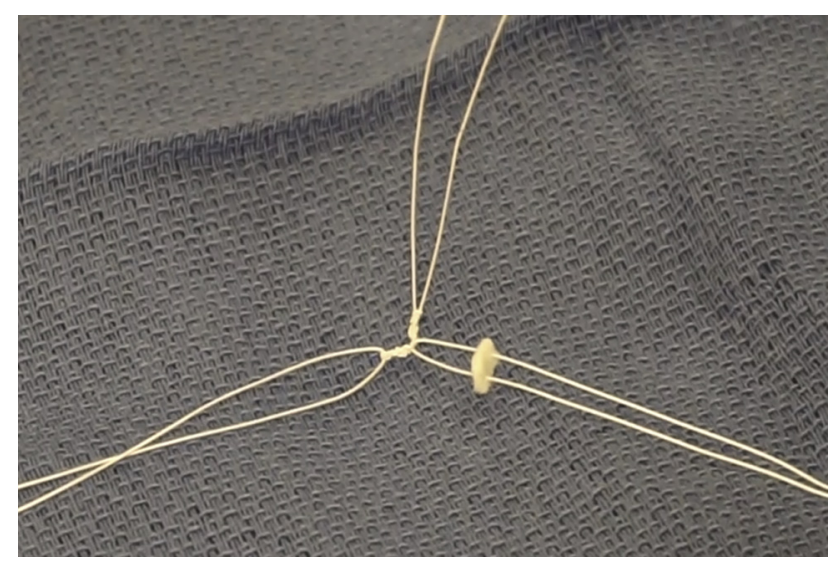

FIGURE 1. A multiloop suture constructed from 2 polytetrafluoroethylene sutures (CV-4 Gore-Tex; WL Gore \& Associates Inc, Newark, Del) tied to a third, which was passed through a felt pledget.
TABLE 1. Comparison of echocardiographic data from preoperation to follow-up

\begin{tabular}{lcc}
\hline \multicolumn{1}{c}{ Variable } & Preoperation & Follow-up \\
\hline LVEDV $(\mathrm{mL})$ & $142 \pm 70$ & $113 \pm 37$ \\
LVESV $(\mathrm{mL})$ & $73 \pm 56$ & $56 \pm 53$ \\
\hline LVEDD $(\mathrm{mm})$ & $60 \pm 11$ & $60 \pm 5$ \\
LVESD $(\mathrm{mm})$ & $42 \pm 10$ & $45 \pm 11$ \\
LVEF\% & $37 \pm 10$ & $49 \pm 26$ \\
WMSI & $1.8 \pm 0.4$ & $1.6 \pm 0.6$ \\
\hline
\end{tabular}

Values are presented as mean \pm standard deviation. $L V E D V$, Left ventricular enddiastolic volume; $L V E S V$, left ventricular end-systolic volume; $L V E D D$, left ventricular end-diastolic diameter; $L V E S D$, Left ventricular end-systolic diameter; $L V E F$, left ventricular ejection fraction; WMSI, wall motion score index.

hospital mortality occurred in this series. At 3 months postoperation, transthoracic echocardiogram revealed good valvular function and no MR recurrence.

\section{DISCUSSION}

IMR is a consequence of global remodeling of the left ventricle, resulting in annular dilatation and papillary muscle displacement. The most common treatment for IMR is RA, to address dilatation, and concomitant CABG surgery. Several PMR approaches have been introduced as an adjunct to improve long-term IMR outcomes.

We present a novel and simplified PMR technique. Unlike the technique pioneered by and colleagues, ${ }^{4}$ pledgets are used to alleviate tension caused by the neochords on the ischemic papillary muscle. Multiple sutures can be added to the loop without increasing the number of passes through the muscle, which could predispose it to further ischemia. ${ }^{5}$ This provides greater flexibility and the possibility of including more sutures for greater stability or redundancy, if necessary. Chordal tension on the PPM is first relieved by RA, followed by relocation to address possible leaflet tethering. A downsized ring is used to minimize the tension required to relocate the PPM. Neochordae are anchored to the annulus in the direction of the jet, where the displacement of the PPM is most dramatic (P2 or P3), to achieve a physiologically consistent relocation. Sutures are marked in sequence and passed through the PPM at an angle and through the annulus at an orientation that avoids potential twisting of the muscle. Biomechanically speaking, this approach has the potential to allow for a more even distribution of tension over a larger surface area of the papillary muscle, as well as over multiple sutures at the level of the neochordae, and can be tailored based on the specific mechanism of MR.

This novel multiloop PMR technique adds further versatility to the original technique. It can be easily included in routine IMR procedures to adjust the subvalvular apparatus, while potentially providing a more durable 

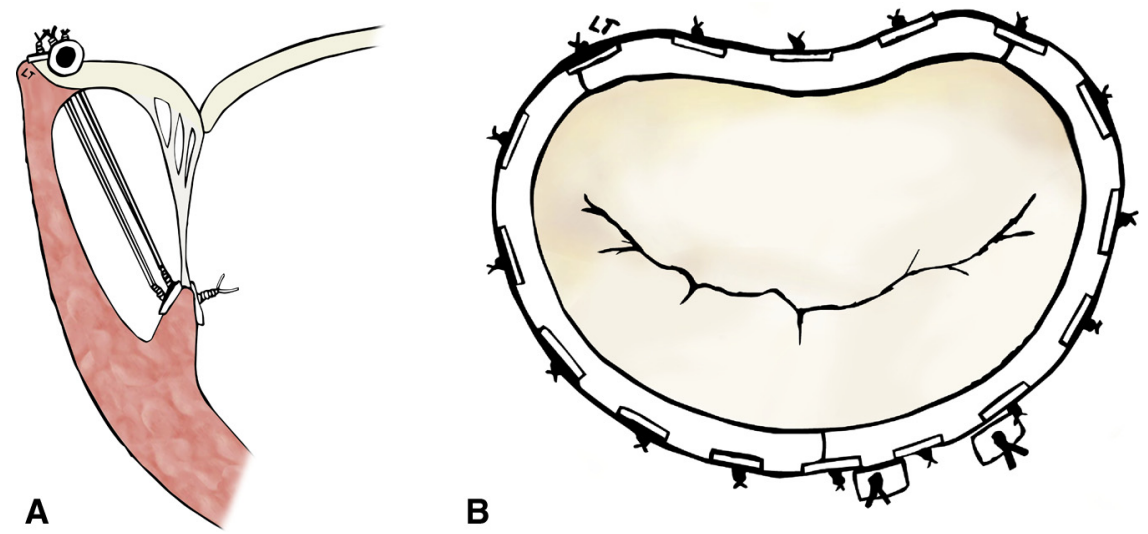

FIGURE 2. A, Diagram of the sagittal view of the multiloop anchored to the posteromedial papillary muscle with neochordae at P2 and P3. B, Superior view.

result. Nevertheless, these preliminary findings warrant further investigation with a larger cohort of patients and longer follow-up to ascertain the benefits in this complex subset of patients.

\section{References}

1. Asgar AW, Mack MJ, Stone GW. Secondary mitral regurgitation in heart failure: pathophysiology, prognosis, and therapeutic considerations. J Am Coll Cardiol. 2015;65:1231-48.
2. Goldstein D, Moskowitz AJ, Gelijns AC, Ailawadi G, Parides MK, Perrault LP, et al. Two-year outcomes of surgical treatment of severe ischemic mitral regurgitation. N Engl J Med. 2015;374:344-53.

3. Fattouch K, Castrovinci S, Murana G, Dioguardi P, Guccione F, Nasso G, et al Papillary muscle relocation and mitral annuloplasty in ischemic mitral valve regurgitation: midterm results. J Thorac Cardiovasc Surg. 2014;148:1947-50.

4. Kron IL, Green R, Cope JT. Surgical relocation of the posterior papillary muscle in chronic ischemic mitral regurgitation. Ann Thorac Surg. 2002;74:600-1.

5. Ibrahim M, Rao C, Athanasiou T. Artificial chordae for degenerative mitral valve disease: critical analysis of current techniques. Interact Cardiovasc Thorac Surg. 2012;15:1019-32. 